\title{
BCL2-938C $>$ A AND CALCA-1786T $>$ C POLYMORPHISMS IN Aseptic Loosened Total Hip Arthroplasty
}

\author{
C. Wedemeyer ${ }^{1}$, M. D. Kauther ${ }^{1}$, S. Hanenkamp ${ }^{1,2}$, H. Nückel ${ }^{3}$, M. Bau ${ }^{2}$, W. Siffert ${ }^{2}$, H. S. Bachmann ${ }^{2}$ \\ ${ }^{1}$ Department of Orthopaedic Surgery, Medical Faculty, ${ }^{2}$ Institute of Pharmacogenetics, Medical Faculty, ${ }^{3}$ Department of Haematology, \\ Medical Faculty, University of Duisburg-Essen, Essen, Germany
}

\begin{abstract}
The search for influencing factors and new pathways in aseptic loosening of arthroplasties is a major focus of recent studies. Analyses of polymorphisms of genes revealed a correlation between a specific allele variant and aseptic loosening. The BCL2 gene encoding $\mathrm{Bcl}-2$ with its BCL2 $-938 \mathrm{C}>\mathrm{A}$ polymorphism is a crucial factor of cell cycle control and cell survival. The CALCA -1786T $>\mathrm{C}$ polymorphism belongs to the CALCA gene encoding alpha-Calcitonin Gene Related Peptide (CGRP) and Calcitonin. Both proteins are important in bone metabolism and capable to influence the process of aseptic loosening. To date, no studies are reported for aseptic loosening with these two single nucleotide polymorphisms (SNPs). In a retrospective study we determined the distribution of the $B C L 2-938 C>A$ and the CALCA-1786T $>$ C polymorphisms in 87 subjects with aseptic loosened hip arthroplasties using RFLP and pyrosequencing analysis. Genotype distribution with prognosis of the hip arthroplasty showed neither an association with clinical characteristics of the patients nor the implantation technique. We were unable to detect any influence of these polymorphisms on time to aseptic loosening.
\end{abstract}

\section{INTRODUCTION}

Aseptic loosening of total joint replacement is one of the major reasons for long term failure of arthroplasty (Silva and Sandell 2002). Various studies have been performed to understand the mechanisms of aseptic loosening. The local osteolysis surrounding the prosthesis is initiated by an aseptic inflammatory response after incorporation of wear particles by macrophages. Via cytokines macrophages stimulate the proliferation and differentiation of precursor osteoclasts into mature osteoclasts and fibroblasts producing a benign local granulomatous tissue. This process results in implant instability requiring expensive revision surgery. We have recently published two articles about the influence of apoptosis (Landgraeber, Toetsch et al. 2006; Landgraeber, von Knoch et al. 2008) and neuropeptides (i.e. alpha - Calcitonin-Gene Related Peptide (CGRP) (Wedemeyer, Neuerburg et al. 2007) in aseptic loosening.

Firstly, apoptosis is an important process to regulate homeostasis in tissue. The proliferation and apoptosis in normal tissue is controlled by a variety of proteins of the Bcl-2 family (Chao and Korsmeyer 1998) con- sisting of different regulators which can block or accelerate the apoptosis (Kroemer 1997). The founding member of this family, Bcl-2 itself, is an inhibitor of apoptotic cell death and can act in both antiapoptotic and antiproliferative manners (Cory and Adams 2002). Recently we showed a slight reaction using Bcl-2 antibodies in immunochemistry with macrophages and giant cells in granulomatous tissue in loosened arthroplasties and concluded that it is most likely that wear debris induced apoptotic reactions (Landgraeber, Toetsch et al. 2006). The BCL2 gene is located on chromosome 18q21.3 (Seto, Jaeger et al. 1988; Young and Korsmeyer 1993). It consists of three exons and two promoters. These two promoters have distinct functions. Activation of the promoter P2 decreases the activity of the other promoter P1, therefore acting as a negative regulatory element (Bredow, Juri et al. 2007). Park et al. have identified a common single nucleotide polymorphism (SNP, -938C > A (rs 2279115)) in the inhibitory P2 promoter of the BCL2 gene (Park, Kim et al. 2004). Nückel et al. showed that this polymorphism has functional properties and alters the promoter activity of P2 and inversely the activity of P1 (Nuckel, Frey et al. 2007). Furthermore this SNP has been repeatedly associated with different diseases (Bachmann, Otterbach et al. 2007; Chen, Hu et al. 2007).

Secondly, a possible influence of the nervous system on particle induced osteolysis was shown by Ahmed et al.. They found alpha-CGRP-immunoreactive nerve fibres at the interface membrane of arthroplasty. Alpha-CGRP is a neurotransmitter produced by the central and peripheral nervous system and can be found in all tissues surrounding joints and close to the epiphyseal plate, indicating that alpha-CGRP has a function in bone remodelling (Irie, Hara-Irie et al. 2002). Our study group recently demonstrated the impact on particle induced osteolysis in alpha-CGRP knock-out mice (Wedemeyer, Neuerburg et al. 2007). Therefore we focused on the CALCA gene (11p15.2p15.1), encoding both alpha-CGRP and Calcitonin. Calcitonin is nearly exclusively produced by thyroid cells (Jacobs, Goodman et al. 1981; Amara, Jonas et al. 1982; Rosenfeld, Mermod et al. 1983). This polypeptide consisting of 32 amino acids plays a role in calcium haemostasis by the inhibition of osteoclasts. However, its physiological function is not clarified in all details (Huebner, Schinke et al. 2006). The CALCA gene consists of one promoter and six exons. $C A L$ $C A$ polymorphisms have been examined in many dif- 
ferent diseases (Shipman, Schraml et al. 1993; Buervenich, Xiang et al. 2001; Hink, Hokanson et al. 2003; Goodman, Ferrell et al. 2005; Morita, Nakayama et al. 2007). Two common polymorphisms - CALCA $1786 \mathrm{~T}>\mathrm{C}(\mathrm{rs} 3781719)$ and $-1752 \mathrm{C}>\mathrm{G}(\mathrm{rs} 1553005)-$ in complete linkage are located in the promoter region. Goodman et al. showed a strong association of these $C A L C A$ polymorphism with the risk of ovarian cancer among some subgroups (Goodman, Ferrell et al. 2005).

The aim of this study was to investigate the possible influence of the functional BCL2 (-938 C>A) and CALCA $(-1786 \mathrm{~T}>\mathrm{C})$ polymorphisms on aseptic loosening of total hip replacement.

\section{Materials And Methods}

\section{Patients}

Our collective consisted of 87 Caucasian patients of German ancestry operated for aseptic loosening of prosthetic hip joints at the Department of Orthopaedic Surgery (Medical Faculty, University of Duisburg-Essen, Germany). For all patients strict inclusion and exclusion criteria were applied. Entry criteria for this study were the clinical, radiological and intra-surgical diagnosis of aseptic loosening after total hip arthroplasty due to primary osteoarthritis. Exclusion criteria were any deep infection or the suspicion of implant infection, traumatic loosening, inflammatory diseases or intake of immunosuppressant agents after total hip arthroplasty in the patient's history. In 36 of 87 cases we performed a revision of the stem and the cup, in 24 of 87 cases only the stem and in 27 cases of 87 only the cup. The present study was performed according to the Declaration of Helsinki and approved by the local Ethics Committee of the University Hospital of Essen.

\section{Determination of BCL2 AND CALCA GENOTYPES}

DNA was extracted from routinely excised granulomatous scared tissue surrounding the prosthesis. Isolation of genomic DNA was performed using a commercially available kit (QIAamp, Qiagen, Hilden, Germany) following the manufacturer's instructions. Genotypes of BCL2 -938C >A were determined by Slowdown PCR and pyrosequencing as previously described (Goodman, Ferrell et al. 2005; Nuckel, Frey et al. 2007). CALCA -1786T $>C$ genotypes were determined by PCR using the following primers: forward primer 5'-CGCTGGGCTGT'TTCTCACAATAT-3'; reverse primer, 5'-GTTAGACAGGAGTTCAATTAC AGTTGGC -3'. After an initial denaturation at $94^{\circ} \mathrm{C}$, 38 cycles of DNA amplification were done using Taq PCR Mastermix (Eppendorf, Hamburg, Germany) at $94^{\circ} \mathrm{C}$ for 45 seconds, $62^{\circ} \mathrm{C}$ for 40 seconds, and $72{ }^{\circ} \mathrm{C}$ for 45 seconds. The $144 \mathrm{bp}$ PCR products were digested using the restriction enzyme BsmAI (NEB, Ipswich, MA) and analyzed on a $2.5 \%$ agarose gel. Unrestricted products (144 bp) represent the TT genotype, completely restricted products $(115+29 \mathrm{bp})$ represent the CC genotype.

\section{STATISTICAL ANALYSis}

Kaplan-Meier plots and the log-rank test were used to retrospectively evaluate the time to loosening, dependent on BCL2 and CALCA genotypes, respectively. Kruskal-Wallis test was used for comparison of non-parametric variables where appropriate. Contingency tables and Pearson's $\chi^{2}$-test were used to compare categorical variables using BCL2 and $C A L C A$ genotypes as indicated. ANOVA was used for comparison of continuous variables. The impact of age, BMI, and BCL2 and CALCA genotypes as prognostic factors for time to aseptic loosening were analysed by univariate Cox regression models. Hazard ratios (HR) and 95\% confidence intervals (95\% CI) were calculated from these Cox regression models. Control for deviation from the Hardy-Weinberg equilibrium was conducted with the public domain program Hardy-Weinberg equilibrium by J. Ott (http://www.genemapping.cn/util.htm). Differences were regarded significant at $\mathrm{p}<0.05$. Statistical analysis was performed using SPSS 15.0 (SPSS, Chicago, IL, USA) and GraphPad Prism 4.0 (GraphPad Software, San Diego, CA, USA).

\section{RESULTS}

In our cohort genotype distributions of BCL2 938C > A (CC:22 CA:49 AA:16) as well as CALCA 1786T $>$ C (TT:42 TC:35 CC:10) were not significantly different from Hardy-Weinberg equilibrium. Clinicopathological characteristics are shown in Tables 1 and 2. Age at the first implantation, age at the revision, gender, body weight, body height, Body Mass Index, Paprosky`s score (Paprosky, Bradford et al. 1994) (a classification system in grades, based on preoperative $\mathrm{x}$-rays of the bone loss surrounding a loosened implant), first cup with or without cement and first stem with or without cement were not associated with BCL2 and CALCA genotypes, respectively.

Neither time to aseptic loosening nor median time to aseptic loosening showed a significant association with BCL2 and CALCA genotypes (Fig. 1). Median time to reimplantation due to aseptic loosening was 101 months (range 1-367) for BCL2 -938 CC homozygous patients, 86 months (range 5-384) for patients heterozygous for BCL2 -938 and 52 months (range 0-344) for GG genotype carriers ( $p=0.543$ ). Kaplan-Meier curve dependent on BCL2 -938 genotypes showed no significant association (Fig. 1A, p = 0.985). Following Hazard Ratios (HR) and 95\% Confidence intervals (CI) were calculated for BCL2 -938 genotypes: CA vs. CC HR 0.982, 95\% CI 0.59-1.63, p $=0.945$ and AA vs. CC HR 1.033, 95\%CI 0.54-1.99, p $=0.923$. Median time to aseptic loosening for $C A L$ $C A-1786 \mathrm{~T}>\mathrm{C}$ genotypes was 69 months (range 2357) for CC genotype carriers, 57 months (range 1384) for heterozygous and 82.5 months (range 0-273) for TT homozygous patients $(\mathrm{p}=0.772)$. No association was detectable in CALCA -1786 dependent Kaplan-Meier curve (Figure 1B, p = 0.378). Hazard Ratios were $0.793,95 \% \mathrm{CI} 0.50-1.26, \mathrm{p}=0.323$ for $\mathrm{TC}$ vs. T'T and HR 0.647, 95\%CI 0.32-1.31, p = 0.224 for CC vs. TT. Additionally performed subgroup analyses 
Table 1. Clinical characteristics and genotype distribution in patients with aseptic loosening.

\begin{tabular}{|c|c|c|c|c|c|}
\hline & \multirow{2}{*}{ All } & \multicolumn{3}{|c|}{ BCL-2 genotype } & \multirow{2}{*}{$\mathrm{p}$-value } \\
\hline & & $\mathrm{CC}$ & $\mathrm{CA}$ & AA & \\
\hline n $(\%)$ & 87 & $22(25.28)$ & $49(56.32)$ & $16(18.4)$ & \\
\hline Age at implantation (y) & $60.18 \pm 13.14$ & $58.14 \pm 14.06$ & $60.94 \pm 13.72$ & $60.69 \pm 10.08$ & 0.703 \\
\hline Age at replantation $(y)$ & $69.31 \pm 10.27$ & $67.41 \pm 12.77$ & $70.04 \pm 9.81$ & $69.69 \pm 7.79$ & 0.605 \\
\hline \multicolumn{6}{|l|}{ Gender } \\
\hline Female $(\%)$ & $60(68.97)$ & $12(20.0)$ & $35(58.33)$ & $13(21.66)$ & 0.182 \\
\hline Male (\%) & $27(31.03)$ & $10(37.04)$ & $14(51.86)$ & $3(0.11)$ & \\
\hline Weight $(\mathrm{kg})$ & $75.11 \pm 17.11$ & $75.88 \pm 19.59$ & $76.1 \pm 16.65$ & $71.13 \pm 15.18$ & 0.592 \\
\hline Height (cm) & $166.80 \pm 8.35$ & $168.09 \pm 6.91$ & $166.77 \pm 9.13$ & $165.13 \pm 7.95$ & 0.563 \\
\hline BMI (kg/m_) & $26.86 \pm 5.07$ & $26.74 \pm 6.04$ & $27.25 \pm 4.92$ & $25.9 \pm 4.15$ & 0.655 \\
\hline \multicolumn{6}{|c|}{ First cup with cement $(\mathrm{n}=83)$} \\
\hline no & $34(40.96)$ & $6(17.65)$ & $19(55.88)$ & $9(26.47)$ & 0.280 \\
\hline yes & $49(59.04)$ & $14(28.57)$ & $28(57.14)$ & $7(14.29)$ & \\
\hline \multicolumn{6}{|c|}{ First stem with cement $(\mathrm{n}=80)$} \\
\hline no & $30(37.5)$ & $4(13.33)$ & $19(63.33)$ & $7(23.33)$ & 0.147 \\
\hline yes & $50(62.5)$ & $16(32.0)$ & $27(54.0)$ & $7(14.0)$ & \\
\hline \multicolumn{6}{|c|}{ Acetabular defects (Paprosky) $n=60$} \\
\hline $\mathrm{I}$ & $6(10)$ & $3(50.0)$ & $2(33.33)$ & $1(16.66)$ & 0.479 \\
\hline II & $23(38.83)$ & $5(21.74)$ & $12(52.17)$ & $3(13.04)$ & \\
\hline III & $31(51.66)$ & $7(22.58)$ & $17(54.84)$ & $7(22.58)$ & \\
\hline \multicolumn{6}{|c|}{ Femoral defects (Paprosky) $n=52$} \\
\hline 1 & $4(7.69)$ & $4(100)$ & 0 & 0 & 0.377 \\
\hline 2 & $23(44.23)$ & $5(21.74)$ & $14(60.87)$ & $4(17.39)$ & \\
\hline 3 & $25(48.08)$ & $8(32.0)$ & $12(48.0)$ & $5(20.0)$ & \\
\hline
\end{tabular}

Data are numbers with percentages given in brackets and numbers with standard deviation, respectively. Categorical variables were analyzed by $\chi^{2}$ statistics. P values were calculated using ANOVA for continuous variables.

Table 2. Clinical characteristics and genotype distribution in patients with aseptic loosening.

\begin{tabular}{|c|c|c|c|c|c|}
\hline & \multirow{2}{*}{ All } & \multicolumn{3}{|c|}{$C A L C A$ genotype } & \multirow{2}{*}{ p-value } \\
\hline & & $\mathrm{CC}$ & $\mathrm{CT}$ & TT & \\
\hline $\mathrm{n}(\%)$ & 87 & $10(11.49)$ & $35(40.22)$ & $42(48.3)$ & \\
\hline Age at implantation (y) & $60.18 \pm 13.14$ & $54.3 \pm 23.17$ & $59.46 \pm 10.47$ & $62.19 \pm 11.85$ & 0.215 \\
\hline Age at replantation $(\mathrm{y})$ & $69.31 \pm 10.27$ & $66.3 \pm 13.27$ & $69.0 \pm 9.74$ & $70.29 \pm 10.02$ & 0.535 \\
\hline \multicolumn{6}{|l|}{ Gender } \\
\hline Female $(\%)$ & $60(68.97)$ & $8(13.33)$ & $27(45.0)$ & $25(41.66)$ & 0.182 \\
\hline Male (\%) & $27(31.03)$ & $2(7.41)$ & $8(29.63)$ & $17(62.96)$ & \\
\hline Weight $(\mathrm{kg})$ & $75.11 \pm 17.11$ & $72.36 \pm 15.92$ & $72.91 \pm 16.83$ & $77.45 \pm 17.61$ & 0.781 \\
\hline Height $(\mathrm{cm})$ & $166.80 \pm 8.35$ & $164.5 \pm 8.17$ & $166.00 \pm 8.31$ & $167.9 \pm 8.45$ & 0.828 \\
\hline BMI (kg/m_) & $26.86 \pm 5.07$ & $26.69 \pm 5.04$ & $26.29 \pm 4.86$ & $27.37 \pm 5.30$ & 0.437 \\
\hline \multicolumn{6}{|c|}{ First cup with cement $(n=83)$} \\
\hline no & $34(40.96)$ & $2(5.88)$ & $17(50.0)$ & $15(44.12)$ & 0.171 \\
\hline yes & $49(59.04)$ & $8(16.33)$ & $16(32.65)$ & $25(51.02)$ & \\
\hline \multicolumn{6}{|c|}{ First stem with cement $(\mathrm{n}=80)$} \\
\hline no & $30(37.5)$ & $2(6.66)$ & $13(43.33)$ & $15(50.0)$ & 0.510 \\
\hline yes & $50(62.5)$ & $7(14.0)$ & $17(34.0)$ & $26(52.0)$ & \\
\hline \multicolumn{6}{|c|}{ Acetabular defects (Paprosky) $n=60$} \\
\hline $\mathrm{I}$ & $6(10)$ & $1(16.66)$ & $2(33.33)$ & $3(50.0)$ & 0.880 \\
\hline II & $23(38.83)$ & $2(8.7)$ & $13(56.52)$ & $8(34.78)$ & \\
\hline III & $31(51.66)$ & $5(16.13)$ & $11(35.48)$ & $15(48.39)$ & \\
\hline \multicolumn{6}{|c|}{ Femoral defects (Paprosky) $\mathrm{n}=52$} \\
\hline 1 & $4(7.69)$ & 0 & $2(50.0)$ & $2(50.0)$ & 0.723 \\
\hline 2 & $23(44.23)$ & $4(17.39)$ & $9(39.13)$ & $10(43.48)$ & \\
\hline 3 & $25(48.08)$ & $2(8.0)$ & $10(40.0)$ & $13(52.0)$ & \\
\hline
\end{tabular}

Data are numbers with percentages given in brackets and numbers with standard deviation, respectively. Categorical variables were analyzed by $\chi^{2}$ statistics. P values were calculated using ANOVA for continuous variables. 
A

BCL2 -938

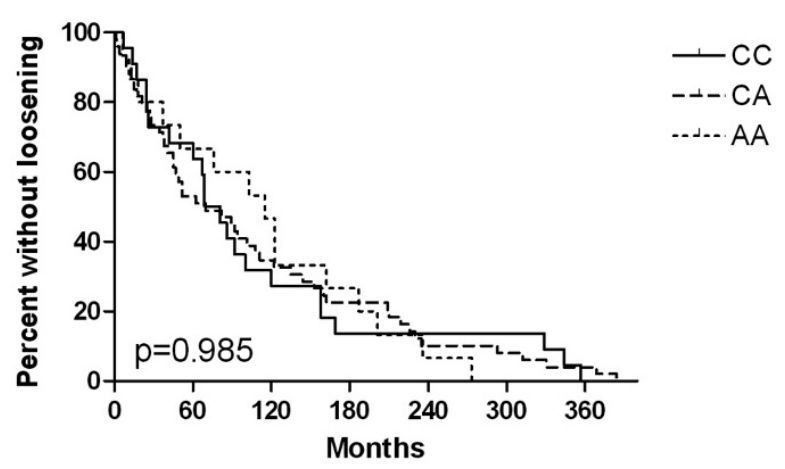

B

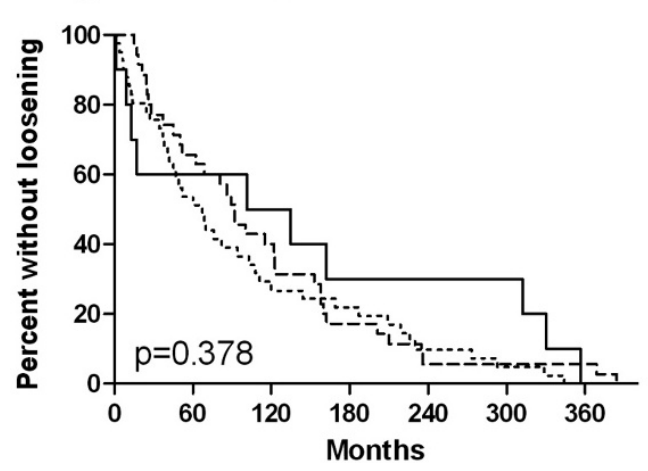

Fig. 1. Time to aseptic loosening based on Kaplan-Meier curves. (A) Patients based on BCL2 -938 genotype. (B) Patients based on $C A L C A-1786$ genotype.

for genotype dependent early aseptic loosening $(\leq 10$ years) and gender differences showed no significant association with genotypes (Data not shown).

\section{Discussion}

Despite multiple studies to understand and treat aseptic loosening, it is still one of the major problems of arthroplasty. The number of potential replacements is limited due to continued loss of bone substance. In contrast to septic loosening or material failure no specific measures are available to reduce the incidence of aseptic implant loosening until now. Several studies aimed to determine risk factors to detect patients at higher risk who may benefit from prophylactic regimens before implantation, e.g. weight reduction, or pharmacological prophylaxis after implantation. More precise prediction of the individual risk would facilitate to make clinical decision. Unfortunately, clinical parameters for aseptic loosening have yielded partly inconclusive results, e.g. age (Berry, Harmsen et al. 2002; Flugsrud, Nordsletten et al. 2007). It is commonly believed that genetic host factors can influence the incidence and course of aseptic loosening. Recent in-vivo studies of our research group showed the genetic influence on aseptic loosening in knock out mice (Wedemeyer, Neuerburg et al. 2007). Therefore, the aim of the present study was to investigate whether a genetic host factor can be found in humans.
The common BCL2 -938 and CALCA -1786 polymorphisms in the genes BCL2 and CALCA may influence time to aseptic loosening in patients with hip joint replacement and could be used as prediction for time of loosening. Firstly, BCL2 was found in the key cells of particle induced osteolysis (macrophages, lymphocytes) in loosened arthroplasties (Landgraeber, Toetsch et al. 2006) and may have an impact in bone metabolism.

Secondly, the CALCA gene is of great importance for the bone metabolism obviously seen in the phaenotype of increased bone formation rate in $C A L C A$ knock out mice. Furthermore we could show higher rates of particle induced osteolysis in alpha-CGRP knock out mice.

All in all, we could not find any significant correlation between time and median time to aseptic loosening and BCL2 -938 as well as CALCA -1786 genotypes. We were not able to compare the genotype distributions of BCL2 -938 and CALCA -1786 with a population of patients with total hip replacement not suffering from aseptic loosening. Therefore we exploratively compared the genotype distributions with a collective of healthy controls witch were comparable with our cohort in age and gender distribution (Nuckel, Frey et al. 2007). Interestingly, BCL2 -938 genotypes showed a significantly different distribution (CC: 21, CA 63, AA 36, p = 0.0416). The BCL2 -938CC genotype was at highest risk for aseptic loosening, Odds Ratio (OR) CC vs. AA 2.357, 95\%CI 1.02-5.46, $\mathrm{p}=0.0434$ with heterozygous at intermediate risk with OR $1.750,95 \%$ CI $0.87-3.52, \mathrm{p}=0.1138$ for CA vs. AA. Although it is too early for conclusions about this association, the detected association would be in line with functional data of this SNP. The A-allele of this polymorphism is associated with increased Bcl-2 expression (Nuckel, Frey et al. 2007) causing less apoptosis around the implant which would explain the significance for less aseptic loosing in patient homozygous and heterozygous for the A-allele of the BCL2 938 SNP. Therefore, this result suggests the BCL2 938 SNP as an interesting candidate for further studies.

Unfortunately, statistic analysis did not show significances for the CALCA SNP in contrast to our previous mammalian study with alpha CGRP knockout mice (Buervenich, Xiang, et al. 2001). On the one hand, this might be mainly due to the control collective and the small number of patients in our study. A larger more homogenous collective might show significant changes and can detect effect from very low penetrance genes. Moreover, conclusions drawn from this study may not be generalised to all patients undergoing hip replacement - because genetic polymorphisms often vary between ethnic groups of patients. On the other hand, it has to be emphasized that the CALCA SNP has shown some significant associations in other studies, but - in contrast to the BCL2 SNP - its functional properties have not been evaluated until now. Caused by our retrospective collective we could not influence slight differences in physical parameters such as implant design, quality of implantation and stress. Aseptic loosening is a multifactorial process caused by foreign body reaction, allergic reaction, particles, cell 
to cell interaction, hydrostatical pressure, micro movement, body weight, failure of implantation and implant (Silva and Sandell 2002; Drees, Eckardt et al. 2007). As different types of arthroplasty show a different extent of osteolysis a genetic analysis should be prospectively undertaken with the same implant type. Asymptomatic patients with the same implant and implantation technique would be a better collective for comparison. Furthermore it can not be ruled out the possibility that other, not yet identified, alterations in genes involved in cell cycle, apoptosis, receptor expression, DNA replication and gene expression may increase the risk of aseptic loosening after total hip replacement.

Therefore prospective, independent studies with strict inclusion criteria have to be undertaken in the future to rule out all factors uncorrelated to genetics. Furthermore, the inclusion criteria for a prospective study should aim at the bone quality. Although, patients with septic and traumatic loosening, rheumatism, intake of immunosuppressants were excluded in our study we did not measure the exact bone quality at implantation and revision, e.g. osteomalacia, osteoporosis, sclerosis and osteopetrosis.

The genetic influence on humans in this multifactorial process could be shown in this analysis.

Until now the outstanding number-one-reason for particle induced osteolysis has not been found and will possibly not be found in the genes either. Definitely, further polymorphisms have to be analysed as a genetic impact on aseptic loosening was obviously and significantly shown in alpha-CGRP knock out mice in our calvarian model. It bears the chance to analyse a genetic impact on particle induced osteolysis.

All in all, this study combined with our results in the mammalian model support the concept of a role of BCL2 -938 genotypes in aseptic loosening. Further independent and prospective replication studies are necessary to confirm the general validity of our findings, and deduced hypotheses and aim at the CALCA -1786 polymorphism for a second time. Additionally, other genes and genetic polymorphisms could play a major role in the interaction of biomaterials and the environment. Research in this field could lead to better understanding of pathomechanisms and secondary improve the results of arthroplasty.

Conflicts of interest: The authors state that they have not any conflicts of interest.

Acknowledgements: The study was supported by the German Research Foundation (DFG), Bonn, Germany (WE 3634/ 1-1).

\section{REFERENCES}

Amara, S. G., V. Jonas, et al. (1982). "Alternative RNA processing in calcitonin gene expression generates mRNAs encoding different polypeptide products." Nature 298(5871): 240-4.

Bachmann, H. S., F. Otterbach, et al. (2007). "The AA genotype of the regulatory $B C L 2$ promoter polymorphism ( $938 \mathrm{C}>\mathrm{A}$ ) is associated with a favorable outcome in lymph node negative invasive breast cancer patients." Clin Cancer Res 13(19): 5790-7.

Berry, D. J., W. S. Harmsen, et al. (2002). "Twenty-five-year survivorship of two thousand consecutive primary Charn- ley total hip replacements: factors affecting survivorship of acetabular and femoral components." J Bone Joint Surg Am 84-A(2): 171-7.

Bredow, S., D. E. Juri, et al. (2007). "Identification of a novel $\mathrm{Bcl}-2$ promoter region that counteracts in a p53-dependent manner the inhibitory P2 region." Gene 404(1-2): 110-6.

Buervenich, S., F. Xiang, et al. (2001). "Identification of four novel polymorphisms in the calcitonin/alpha-CGRP $(C A L C A)$ gene and an investigation of their possible associations with Parkinson disease, schizophrenia, and manic depression." Hum Mutat 17(5): 435-6.

Chao, D. T. and S. J. Korsmeyer (1998). "BCL-2 family: regulators of cell death." Annu Rev Immunol 16: 395-419.

Chen, K., Z. Hu, et al. (2007). "Single-nucleotide polymorphisms at the TP53-binding or responsive promoter regions of $\mathrm{BAX}$ and $B C L 2$ genes and risk of squamous cell carcinoma of the head and neck." Carcinogenesis 28(9): 2008-12.

Cory, S. and J. M. Adams (2002). "The Bcl2 family: regulators of the cellular life-or-death switch." Nat Rev Cancer 2(9): 647-56.

Drees, P., A. Eckardt, et al. (2007). "Mechanisms of disease: Molecular insights into aseptic loosening of orthopedic implants." Nat Clin Pract Rheumatol 3(3): 165-71.

Flugsrud, G. B., L. Nordsletten, et al. (2007). "The effect of middle-age body weight and physical activity on the risk of early revision hip arthroplasty: a cohort study of 1,535 individuals." Acta Orthop 78(1): 99-107.

Goodman, M. T., R. Ferrell, et al. (2005). "Calcitonin gene polymorphism CALCA-624 (T/C) and ovarian cancer." Environ Mol Mutagen 46(1): 53-8.

Hink, R. L., J. E. Hokanson, et al. (2003). "Investigation of DUSP8 and CALCA in alcohol dependence." Addict Biol 8(3): 305-12.

Huebner, A. K., T. Schinke, et al. (2006). "Calcitonin deficiency in mice progressively results in high bone turnover." J Bone Miner Res 21(12): 1924-34.

Irie, K., F. Hara-Irie, et al. (2002). "Calcitonin gene-related peptide (CGRP)-containing nerve fibers in bone tissue and their involvement in bone remodeling." Microsc Res Tech 58(2): 85-90.

Jacobs, J. W., R. H. Goodman, et al. (1981). "Calcitonin messenger RNA encodes multiple polypeptides in a single precursor." Science 213(4506): 457-9.

Kroemer, G. (1997). "The proto-oncogene Bcl-2 and its role in regulating apoptosis." Nat Med 3(6): 614-20.

Landgraeber, S., M. Toetsch, et al. (2006). "Over-expression of $\mathrm{p} 53 / \mathrm{BAK}$ in aseptic loosening after total hip replacement." Biomaterials 27(15): 3010-20.

Landgraeber, S., M. von Knoch, et al. (2008). "Extrinsic and intrinsic pathways of apoptosis in aseptic loosening after total hip replacement." Biomaterials 29(24-25): 3444-3450.

Morita, A., T. Nakayama, et al. (2007). "Association between the calcitonin-related peptide alpha $(C A L C A)$ gene and essential hypertension in Japanese subjects." Am J Hypertens 20(5): 527-32.

Nuckel, H., U. H. Frey, et al. (2007). "Association of a novel regulatory polymorphism $(-938 \mathrm{C}>\mathrm{A})$ in the BCL2 gene promoter with disease progression and survival in chronic lymphocytic leukemia." Blood 109(1): 290-7.

Paprosky, W. G., M. S. Bradford, et al. (1994). "Classification of bone defects in failed prostheses." Chir Organi Mov 79(4): 285-91.

Park, B. L., L. H. Kim, et al. (2004). "Identification of variants in cyclin D1 (CCND1) and B-Cell CLL/lymphoma 2 (BCL2)." J Hum Genet 49(8): 449-54.

Rosenfeld, M. G., J. J. Mermod, et al. (1983). "Production of a novel neuropeptide encoded by the calcitonin gene via tissue-specific RNA processing." Nature 304(5922): 129-35. 
Seto, M., U. Jaeger, et al. (1988). "Alternative promoters and exons, somatic mutation and deregulation of the Bcl-2-Ig fusion gene in lymphoma." Embo J 7(1): 123-31.

Shipman, R., P. Schraml, et al. (1993). "Loss of heterozygosity on chromosome $11 \mathrm{p} 13$ in primary bladder carcinoma." Hum Genet 91(5): 455-8.

Silva, M. J. and L. J. Sandell (2002). "What's new in orthopaedic research." J Bone Joint Surg Am 84-A(8): 14906.

Wedemeyer, C., C. Neuerburg, et al. (2007). "Polyethylene particle-induced bone resorption in alpha-calcitonin generelated peptide-deficient mice." J Bone Miner Res 22(7): 1011-9.

Young, R. L. and S. J. Korsmeyer (1993). "A negative regulatory element in the bcl-2 5 -untranslated region inhibits expression from an upstream promoter." Mol Cell Biol 13(6): 3686-97.
Received: October 27, 2008 / Accepted: March 31, 2009

Address for correspondence:

Dr. med. Christian Wedemeyer

Department of Orthopaedics,

University of Duisburg-Essen

Pattbergstr. 1-3

45239 Essen

Germany

Phone +49(0)201/4089-0

Fax +49(0)201/4089-2722

E-mail: christian.wedemeyer@uni-duisburg-essen.de 